\title{
PHEOCHROMOCYTOMA - THE RARE REASON OF CUSHING'S SYNDROME DUE TO ECTOPIC CORTICOTROPIN SECRETION.
}

Authors: Elwira Przybylik-Mazurek ${ }^{1}$, Ewelina Rzepka ${ }^{1}$, Anna Kurzyńska ${ }^{1}$, Alicja Hubalewska- Dydejczyk ${ }^{1}$, Anna Sowa- Staszczak ${ }^{1}$ Andrzej Budzyński², Magdalena Białas ${ }^{3}$, Robert Chrzan ${ }^{4}$.

Department of Endocrinology Medical College Jagiellonian University Krakow, ${ }^{2}$ II Chair of Surgery Medical College Jagiellonian University Krakow, ${ }^{3}$ Chair of Pathomorphology Medical College Jagiellonian University Krakow, ${ }^{4}$ Chair of Radiology Medical College Jagiellonian University Krakow.

\section{OBJECTIVES}

Cushing syndrome due to ectopic (adrenocorticotropic hormone) ACTH secretion (EAS) constitutes approximately $10 \%$ of Cushing's syndrome (CS). In this group only in about $5 \%$ cases pheochromocytoma is the source of ACTH.

\section{CASE 1}

A 70 year-old woman with 3-months history of malaise, weakness, abdominal pain, loss of weight and appetite, hypertension and diabetes mellitus. One month earlier she had an episode of acute renal insufficiency after coronarography. Patient revealed mental confusion, cachexia, swelling of legs, skin with increased pigmentation, echymoses and petechiae. The laboratory data showed elevated leukocytosis, hyperglycemia, severe hypokalemia, and metabolic alkalosis (pH 7.661 [7.380 - 7.420], pCO2 34.7 $\mathrm{mmHg}$ [32.5 - 43.7], pO2 $42.2 \mathrm{mmHg}$ [75.0 - 95.0], HCO3 $38.3 \mathrm{mmol} / \mathrm{l}$ [22.0 26.0], BE $16.7 \mathrm{mmol} / /[-1.0$ - 3.0]), markedly elevated serum cortisol concentration and ACTH without cortisol suppression after dexamethasone. Measurements of Metanephrine and Normetanephrine in urine were increased. MRI and somatostatin receptor scintigraphy (SRS) disclosed 40 $\mathrm{mm}$ mass in right adrenal gland. After pharmacological treatment patient underwent laparoscopic surgery. Histological examinations confirmed EAS and pheochromocytoma. Unfortunately in the 9-th day after surgery patient died because of ARDS syndrome.

\begin{tabular}{|c|c|c|c|c|c|c|c|c|}
\hline parameter & $\begin{array}{l}\text { WBC } \\
10^{\prime} 3 / \mathrm{ul} \\
{[4.00-10.00]}\end{array}$ & $\begin{array}{l}\text { Potassium } \\
\mathrm{mmol} / \mathrm{l} \\
{[3.5-5.1]}\end{array}$ & $\begin{array}{l}\text { Sodium } \\
\text { mmol/l } \\
{[136.0-145.0]}\end{array}$ & $\begin{array}{l}\text { ALT } \\
\text { U/I } \\
{[5-33]}\end{array}$ & \begin{tabular}{|l|} 
GGTP \\
U/I \\
{$[5-36]$}
\end{tabular} & \begin{tabular}{|l} 
Ch-E \\
U/l \\
{$[5320-$} \\
$12920]$ \\
\end{tabular} & $\begin{array}{l}\text { Urea } \\
\mathrm{mmol} / \mathrm{l} \\
{[2.76-8.07]}\end{array}$ & $\begin{array}{l}\text { Creatynine } \\
\text { Umol// } \\
{[44-80]}\end{array}$ \\
\hline value & 16.99 & $\begin{array}{l}2.4-2.9- \\
4.3\end{array}$ & $134.0-143.0$ & 128 & 115 & 2537 & 5.8 & 75.0 \\
\hline parameter & $\begin{array}{l}\text { Morning } \\
\text { cortisol } \\
\text { ug/dl } \\
{[2.3-20.9]}\end{array}$ & $\begin{array}{l}\text { Midnight } \\
\text { cortisol } \\
\text { ug/dl } \\
\text { [2.3- 20.9] }\end{array}$ & $\begin{array}{l}\text { ACTH } \\
\mathrm{pg} / \mathrm{ml} \\
{[6.0-56.1]}\end{array}$ & $\begin{array}{l}\text { VMA } \\
\text { umol/24h } \\
\text { [9-35] }\end{array}$ & \multicolumn{2}{|c|}{$\begin{array}{l}\text { MN } \\
\text { ug/24h } \\
\text { [52-341] }\end{array}$} & $\begin{array}{l}\text { NMN } \\
\text { ug/24h } \\
{[88-440]}\end{array}$ & $\begin{array}{l}\mathrm{CgA} \\
\mathrm{nmol} / \mathrm{I} \\
{[0.00-6.00]}\end{array}$ \\
\hline value & $79-80$ & $70-78$ & $360-376$ & 135.2 & \multicolumn{2}{|c|}{2997.2} & 1928.3 & 58.6 \\
\hline
\end{tabular}
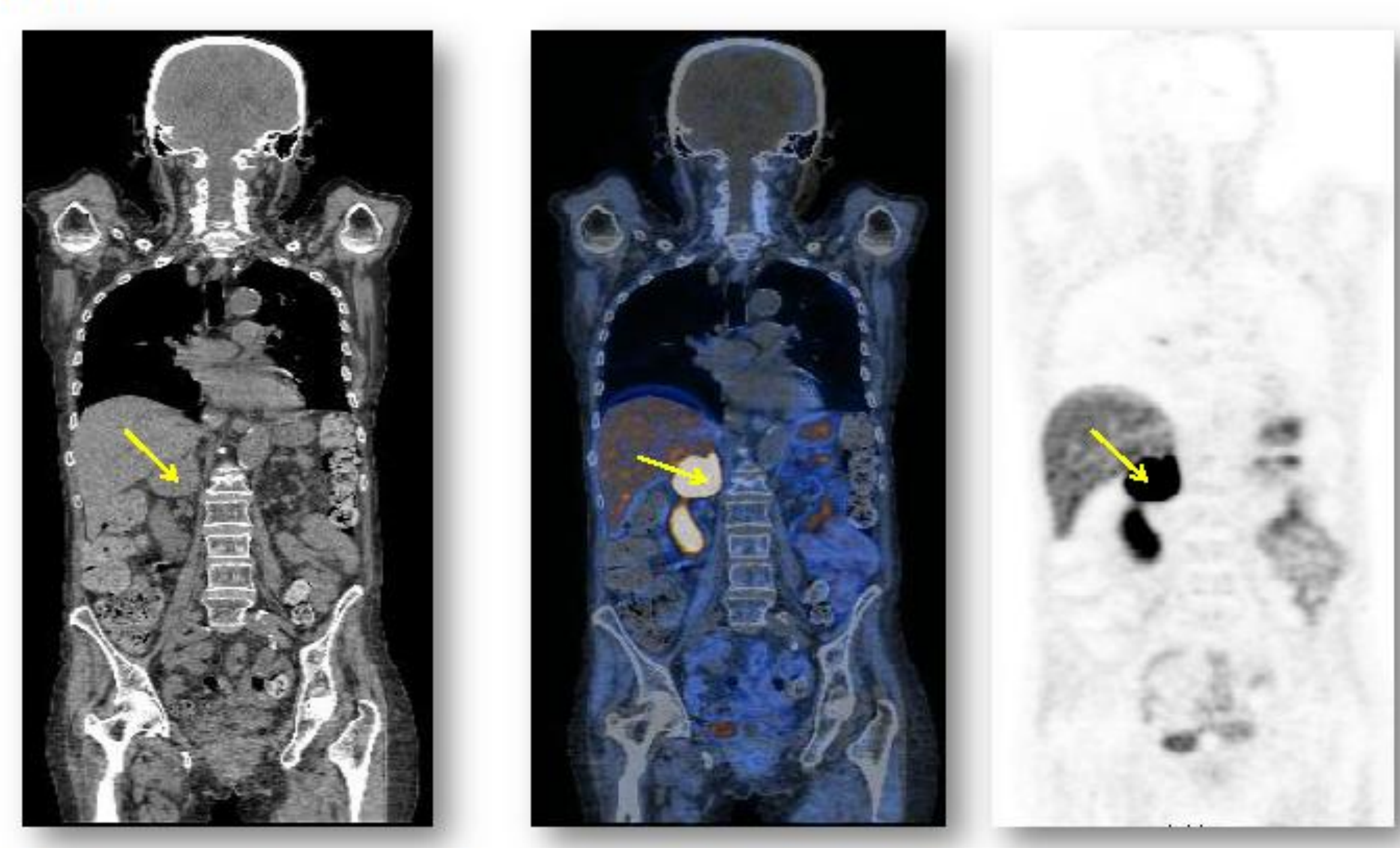

Fig.1. Scintigraphy examination with Ga-68-labelled somatostatin analogues.

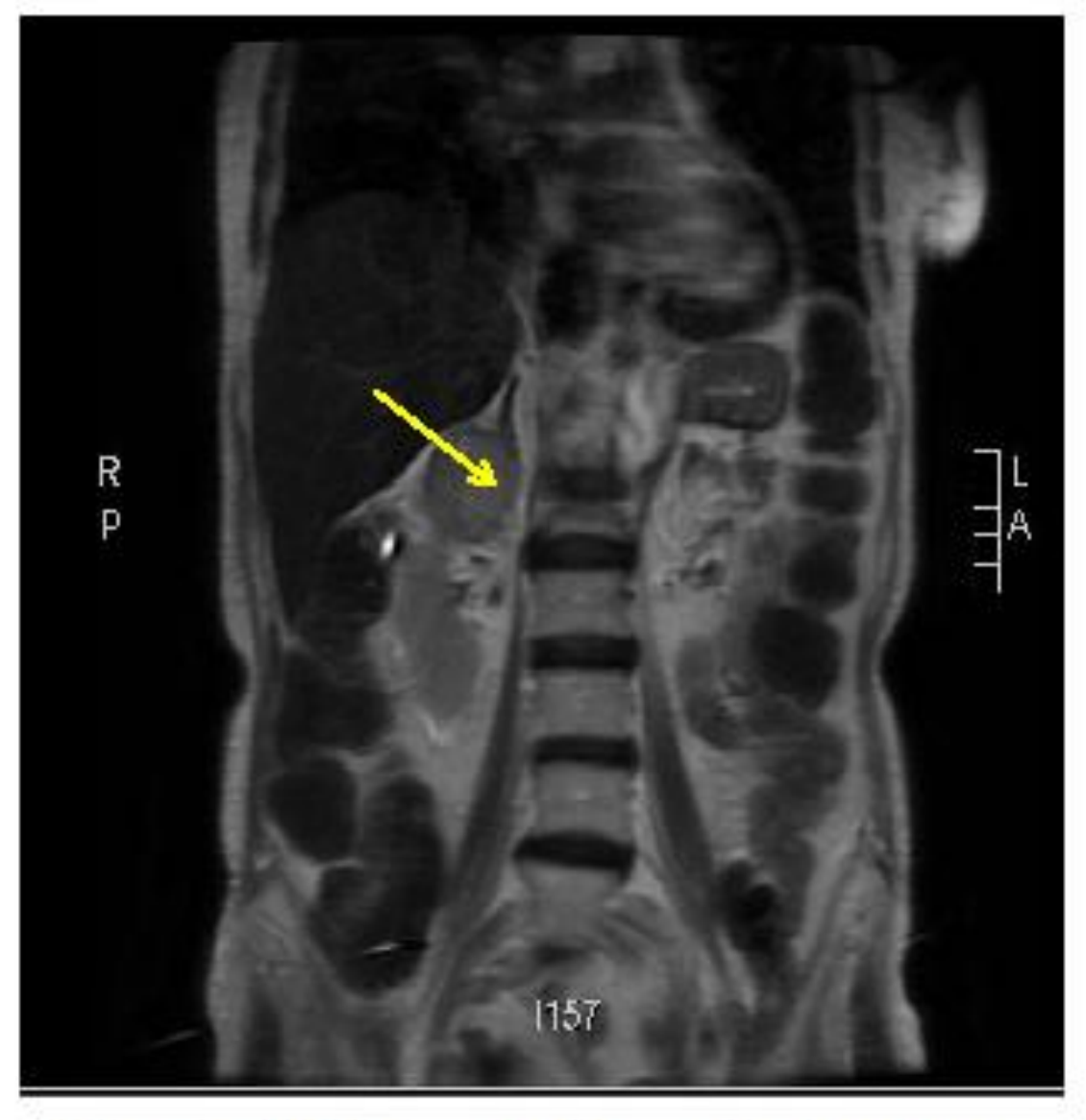

Fig.2. MRI of the abdomen.

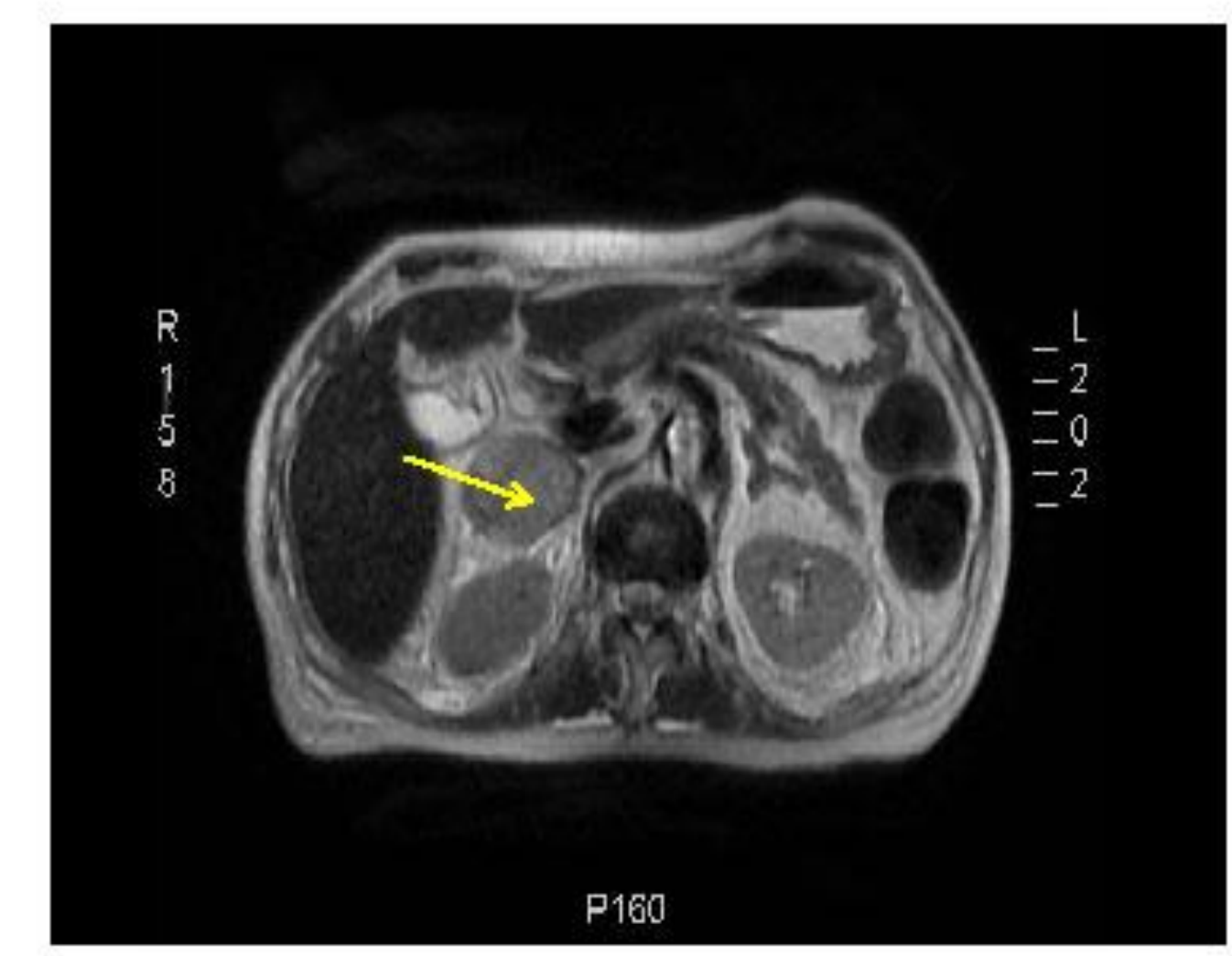

Fig. 3. MRI of the abdomen.

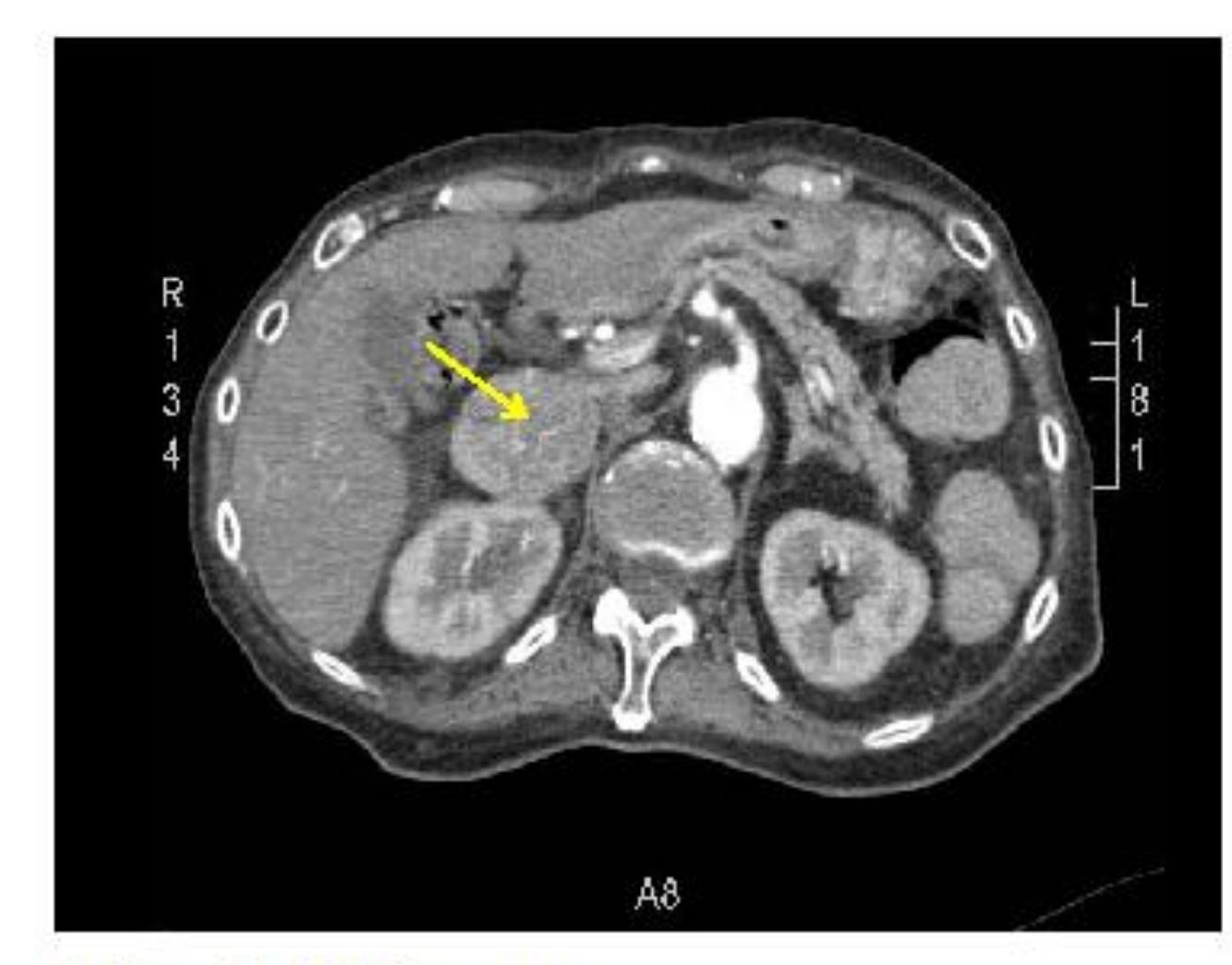

Fig.4. CT scan

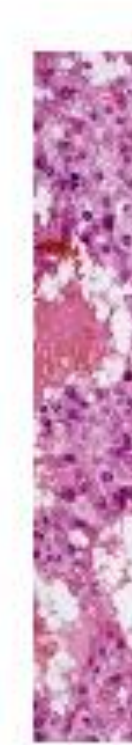
A: HE, B: Chromogranin- A, C: reaction to ACTH, D melan-A

\section{CONCLUSIONS}

EAS is difficult to diagnose. Other organs lesions make the prognosis worse.

\section{METHODS}

We present two clinical cases of patients with EAS by pheochromocytoma.

\section{CASE 2}

A 61 year-old woman with 2-months history of weight gain, proximal myopathy, depressive disorders, abdominal pain, diabetes mellitus of recent onset and worseness of hypertension control. Patient had facial and leg edema, plethoric face, dermal and muscle atrophy and moderate central obesity. The laboratory data showed hyperglycemia, moderate hypokalemia, moderate leukocytosis, disturbed circadian cortisol rhythm and increased ACTH level. Measurements of Metanephrine and Normetanephrine in urine were increased. Abdominal CT scan and SRS revealed $30 \mathrm{~mm}$ mass in right adrenal gland and radiological signs suggested pheochromocytoma. After pharmacological treatment patient was successfully laparoscopic operated and all signs of Cushing's syndrome regressed. Histological examinations confirmed EAS and pheochromocytoma.

\begin{tabular}{|c|c|c|c|c|c|c|c|c|c|c|}
\hline parameter & \multicolumn{2}{|c|}{$\begin{array}{l}\text { WBC } \\
10^{\prime} 3 / \mathrm{ul} \\
{[4.00-10.00]}\end{array}$} & \multicolumn{2}{|c|}{$\begin{array}{l}\text { Potassium } \\
\mathrm{mmol} / \mathrm{l} \\
{[3.5-5.1]}\end{array}$} & \multicolumn{2}{|c|}{$\begin{array}{l}\text { Sodium } \\
\text { mmol/l } \\
{[136.0-145.0]}\end{array}$} & \multicolumn{2}{|c|}{$\begin{array}{l}\text { ALT } \\
\text { U/I } \\
{[5-33]}\end{array}$} & $\begin{array}{l}\text { Urea } \\
\text { mmol// } \\
{[2.76-8.07]}\end{array}$ & $\begin{array}{l}\text { Creatynine } \\
\text { Umol// } \\
\text { [44-80] }\end{array}$ \\
\hline value & 15.58 & & 3.6 & & 143.0 & & 68 & & 6.0 & 68.0 \\
\hline parameter & $\begin{array}{l}\text { Morning } \\
\text { cortisol } \\
\text { ug/dl } \\
\text { [ 2.3- 23.3] }\end{array}$ & $\begin{array}{l}\text { Mic } \\
\text { col } \\
\text { ug } \\
\text { [ } 2 \text {. }\end{array}$ & $\begin{array}{l}\text { Inight } \\
\text { tisol } \\
\text { dl } \\
\text { 3- 20.9] }\end{array}$ & & $\begin{array}{l}\text { TH } \\
\mathrm{ml} \\
-56.0]\end{array}$ & $\begin{array}{l}5 \mathrm{HIA} \\
\text { umol/2 } \\
10.0-2\end{array}$ & $\begin{array}{l}4 \mathrm{~h} \\
40.0]\end{array}$ & \begin{tabular}{|l}
$\mathrm{MN}$ \\
$\mathrm{ug} / 24 \mathrm{~h}$ \\
{$[52-341]$}
\end{tabular} & \begin{tabular}{|l|} 
NMN \\
ug/24h \\
[88-440]
\end{tabular} & \begin{tabular}{|l} 
CgA \\
nmol/l \\
{$[6.00-98.00]$}
\end{tabular} \\
\hline value & 32.5 & 25. & & 18 & & 69.1 & & 2490 & 2526.8 & 605 \\
\hline
\end{tabular}

Tab.2. Laboratory results.

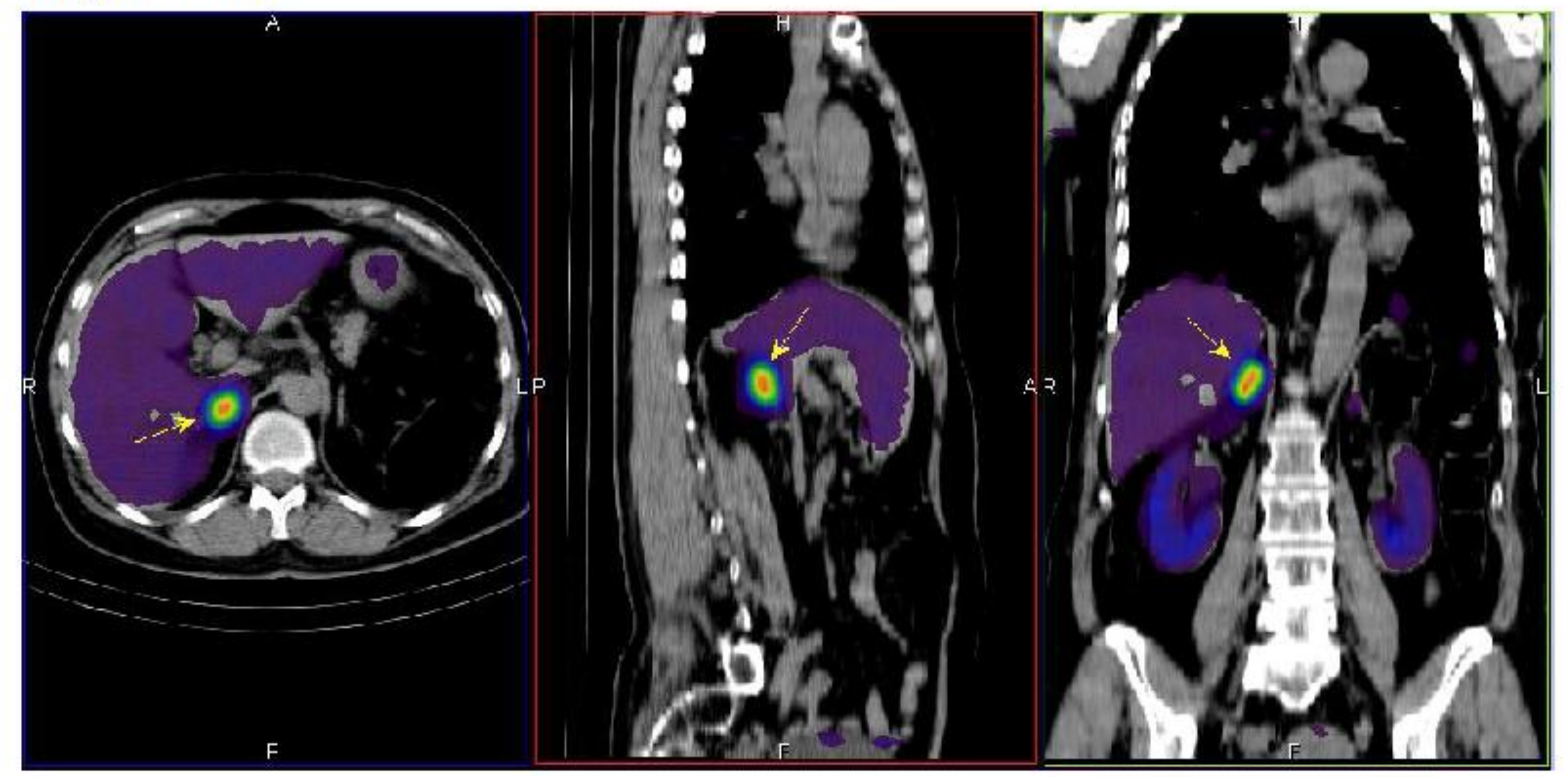

Fig.6. Scintigraphy examination with 99mTc-labelled somatostatin analogues.
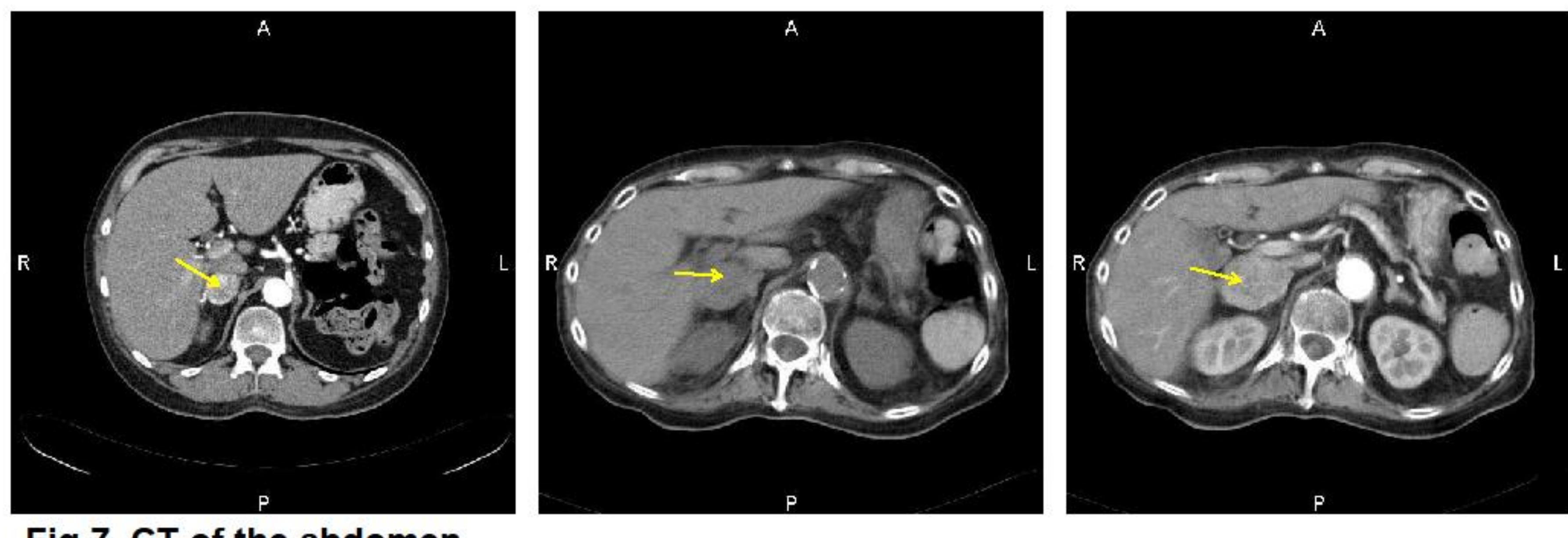

Fig.7. CT of the abdomen.
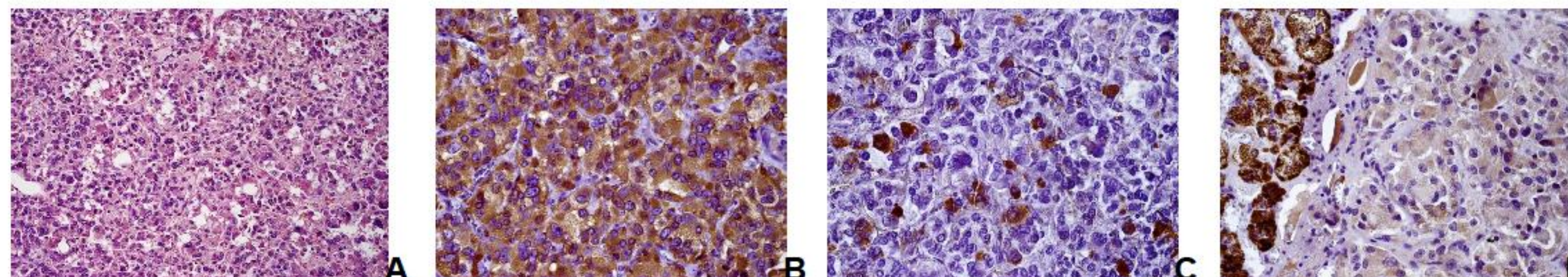

Fig.8. Histological examinations.

A: HE, B: Chromogranin- A, C: reaction to ACTH, D melan-A

\section{References}

Nijhorf MF, Dekkers OM, Vleming LJ, Smit JW, Romijn JA, Pereira AM. ACTH-producing pheochromocytoma: clinical considerations and concise review of the

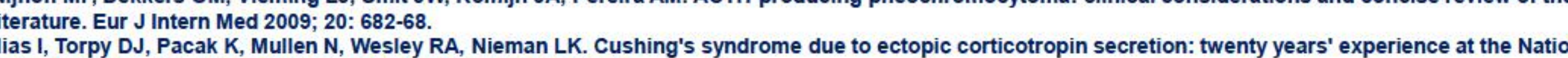

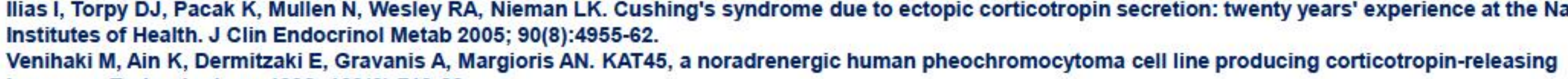

